Boise State University

ScholarWorks

9-7-2009

\title{
Estimating Debye Parameters from GPR Reflection Data Using Spectral Ratios
}

John H. Bradford

Boise State University 


\title{
ESTIMATING DEBYE PARAMETERS FROM GPR REFLECTION DATA USING SPECTRAL RATIOS
}

\author{
John H. Bradford, CGISS, Boise State University, Boise, ID
}

\begin{abstract}
In the GPR frequency range, electromagnetic wave attenuation is largely controlled by dielectric relaxation processes. A primary relaxation commonly occurs in the $10-100 \mathrm{MHz}$ range for many earth materials in which the GPR signal propagates effectively. This relaxation leads to strong nonlinearity in the frequency dependent attenuation and occurs in a frequency range that is often used for groundwater investigations. This non-linearity complicates data analysis but also may provide additional material property information. I investigate inversion for Debye relaxation parameters directly from GPR reflection data, including increasing the bandwidth of the signal by summing the response from $25 \mathrm{MHz}, 50 \mathrm{MHz}, 100 \mathrm{MHz}$, and $200 \mathrm{MHz}$ radar antennas. I compute the timefrequency distribution using spectral decomposition, then use the method of spectral ratios to measure the attenuation vs frequency curve for significant reflection events. I then fit the curve with the multiparameter Debye model. Using synthetic and field data I show that this approach provides reliable estimates of the primary relaxation time for a variety of realistic subsurface models. This approach has the potential to improve our understanding of aquifer material properties.
\end{abstract}

\section{Introduction}

Propagating electromagnetic waves are subject to frequency dependent attenuation which depends on the effective conductivity. The effective conductivity is a function of the real component of the electric conductivity and the complex component of the dielectric permittivity. Turner and Siggins (1994) showed empirically that the frequency dependence of attenuation is approximately linear over the bandwidth of a typical GPR signal with the slope characterized by a frequency independent $\mathrm{Q}^{*}$ parameter that is related to the quality factor. Bradford (2007) derived the a functional form for $\mathrm{Q}^{*}$ that can be used to calculate its value from an arbitrary petrophysical relationship.

Despite Turner's earlier work, development and evaluation of frequency-dependent attenuation analysis for surface-based reflection data remains limited. A few authors have addressed the problem, for example Turner (1994) and Irving and Knight (2003) describe methods for estimating $\mathrm{Q}^{*}$ and applying a deconvolution filter to GPR data to compensate for frequency dependent losses. Other studies suggest the potential of using reflection attenuation analysis for direct material property characterization (Bradford, 1999; Bradford, 2007; Cai and McMechan, 1999; Liu et al., 1998) but further work is needed. It is important to recognize that scattering can result in frequency dependent attenuation (Morlet et al., 1982) that has the same form as intrinsic attenuation and that the attenuation we measure in field data is a combined effect of scattering and intrinsic attenuation.

Here, I investigate determination of the non-linear broadband attenuation of GPR data. I first review the method of spectral ratios. I then employ spectral ratios to measure the non-linear attenuation in synthetic and field data and model this attenuation response using the Debye relaxation model. 


\section{Methodology}

\section{The Spectral Ratio Method}

The amplitude spectrum of an electromagnetic wave after propagation through a material for some distance $r$ is given by

$$
A(r, \omega)=K A_{R}(\omega) e^{-\alpha\left(r-r_{0}\right)}
$$

where $\omega$ is the angular frequency, $A_{R}$ is the amplitude spectrum at the reference location, $r_{0}, \alpha$ is the frequency dependent reflection coefficient, and $\mathrm{K}$ includes all other processes that may impact the amplitude such as transmission losses, reflection coefficient, and geometrical spreading.

With the low loss approximation, the attenuation coefficient is given by (Bradford, 2007)

$$
\alpha \approx \frac{\left[\sigma_{d c}+\varepsilon^{\prime \prime}(\omega) \omega\right]}{2} \sqrt{\frac{\mu_{0}}{\varepsilon^{\prime}(\omega)}},
$$

where $\sigma_{\mathrm{dc}}$ is the DC electric conductivity, $\varepsilon^{\prime}$ and $\varepsilon^{\prime \prime}$ is are the real and imaginary components of the complex dielectric permittivity respectively, and $\mu_{0}$ is the magnetic permeability of free space (here I have assumed non-magnetic material). For some materials, a Debye relaxation model (Debye, 1945), or a superposition of Debye models (Xu and McMechan, 1997) can provide a description of relaxation processes that occur within the GPR frequency band. The Debye model gives the complex dielectric permittivity as

$$
\varepsilon^{\prime}-i \varepsilon^{\prime \prime}=\varepsilon_{\infty}+\frac{\varepsilon_{d c}-\varepsilon_{\infty}}{1+i \omega \tau}
$$

where $\varepsilon_{\mathrm{dc}}$ and $\varepsilon_{\infty}$ are the relative DC and high frequency dielectric permittivities respectively, and $\tau$ is the relaxation time.

To apply the spectral ratio method, divide Equation 1 by $A_{R}$ then solve for $\alpha$ to yield

$$
\alpha=\frac{\ln \left(\frac{A_{R}}{A}\right)}{v \Delta t}+\ln (K) .
$$

The distance traveled has been replaced with the velocity $(\mathrm{v})$ times the time interval $(\Delta \mathrm{t})$. The frequency dependent attenuation coefficient is found by fitting a functional form for $\alpha$ to the frequency dependent data given by the right hand side of Equation 4. For the case of Debye relaxation, this requires fitting Equation 4 with the non-linear expression for attenuation given by Equations 2 and 3. Typically, it is assumed that $\mathrm{K}$ is a lumped parameter that includes all frequency independent terms that alter the amplitude. If reflection, transmission, and antenna radiation are frequency independent then the method of spectral ratios allows separation of these effects from the frequency dependent attenuation terms.

In a layered medium, one can use the reflection spectra from two interfaces, with the upper reflection giving $A_{R}$ and the lower reflection giving $A$, to estimate the effective attenuation coefficient in the interval between the two horizons (Bradford, 1999). 


\section{Sensitivity Of The Attenuation Coefficient To The Debye Parameters And Conductivity}

The sensitivity of the frequency dependence of $\alpha$ to the four parameters that describe Equations 2 and $3\left(\varepsilon_{\mathrm{dc}}, \varepsilon_{\infty}, \sigma_{\mathrm{dc}}, \tau\right)$ is best illustrated by examining $\mathrm{d} \alpha / \mathrm{d} \omega$ as a function of each parameter. Here, I begin with base case parameters of $\varepsilon_{\mathrm{dc}}=19, \varepsilon_{\infty}=17, \sigma_{\mathrm{dc}}=1 \mathrm{mS} / \mathrm{m}, \tau=17 \mathrm{~ns}$ then vary each parameter independently (Figure 1).

The gradient of $\alpha$ is strongly dependent on $\varepsilon_{\mathrm{dc}}$ and $\varepsilon_{\infty}$ (Figures 1A and 1B) and these two parameters are highly correlated in determining frequency dependence of the attenuation coefficient. Additionally, the magnitude of $\alpha$ depends strongly on the difference between $\varepsilon_{\mathrm{dc}}$ and $\varepsilon_{\infty}$ and is therefore correlated with the intercept term K. Note that the frequency at the maximum gradient is independent of either $\varepsilon_{\mathrm{dc}}$ or $\varepsilon_{\infty}$. The gradient maxima, however, is determined by relaxation time, $\tau$ (Figure 1C), while the magnitude of the gradient is only a weak function of $\tau$. The relaxation time is the primary control on the non-linearity of the attenuation coefficient and is only weakly correlated with the other three parameters. The gradient of $\alpha$ is weakly dependent on $\sigma_{\mathrm{dc}}$, and the gradient maximum is independent of $\sigma_{\mathrm{dc}}$ (Figure 1D). The magnitude of $\alpha$ however is strongly dependent on $\sigma_{\mathrm{dc}}$. Changes in $\sigma_{\mathrm{dc}}$ primarily result in bulk static shifts of $\alpha$. Therefore, $\sigma_{\mathrm{dc}}$ is strongly correlated with the intercept term $\mathrm{K}$ and estimation of $\sigma_{\mathrm{dc}}$ requires complete knowledge of all terms in $\mathrm{K}$ which include reflection and transmission coefficients.

Given the above observations, it is clear that all four parameters cannot be uniquely determined from the spectral ratios alone. With estimates of either $\varepsilon_{\mathrm{dc}}$ or $\varepsilon_{\infty}$ and $\sigma_{\mathrm{dc}}$ from other measurements, it should be possible to determine the other two parameters $\left(\tau\right.$ and $\varepsilon_{\mathrm{dc}}$ or $\left.\varepsilon_{\infty}\right)$. Also, it is critical that the signal spectrum spans the relaxation frequency and maximizing the bandwidth will better constrain the data fit. For all examples given below, I assume a single Debye relaxation mechanism, hold $\sigma_{\mathrm{dc}}$ and $\varepsilon_{\infty}$ constant, and estimate the parameters $\tau$ and $\varepsilon_{\mathrm{dc}}$ using the Nelder-Mead simplex method (Nelder and Mead, 1965) for non-linear, multi-parameter estimation.
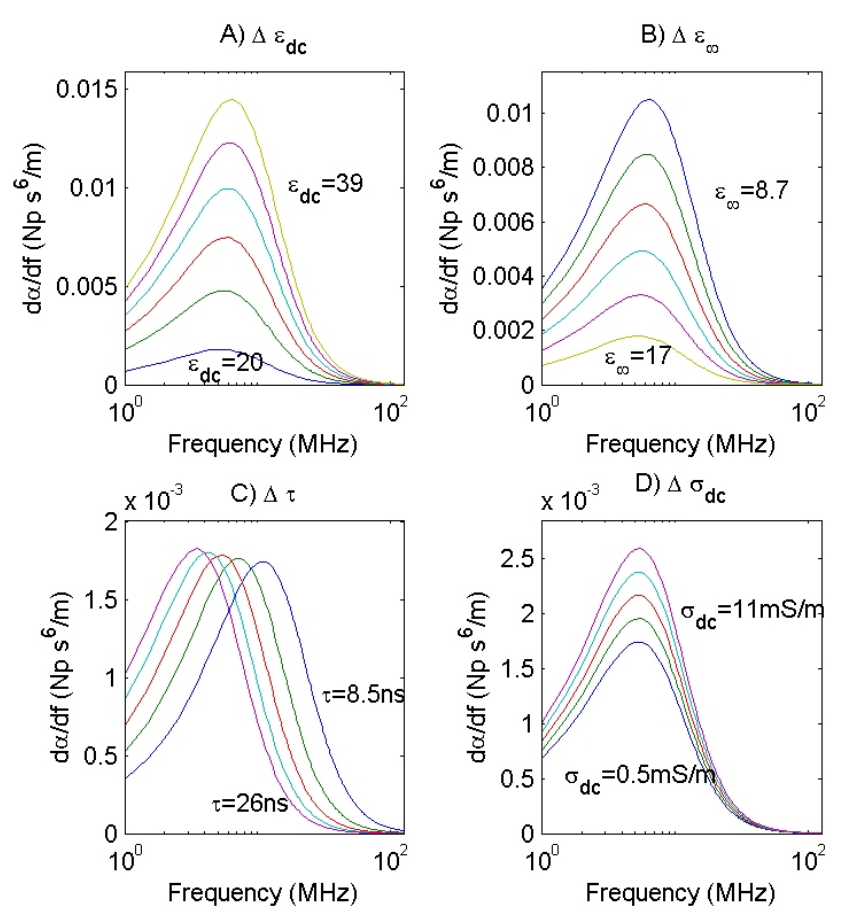

Figure 1: Variation of the gradient in attenuation as a function of frequency for each of the parameters that characterize the Debye relaxation model.

\section{Synthetic Example}

To illustrate the spectral ratio method and to test the sensitivity of the inversion procedure to noise and small scale scattering, I begin with the parameters for a four layer model given in Table 1. The model Debye parameters for layers 1-3 are representative of values found in the literature for unconsolidated saturated sands with varying porosity and layer 4 is representative of clay. I simulate zero offset GPR data using a reflectivity algorithm formulated for frequency dependent material properties, and a Ricker source wavelet (Figure 2). I generate four traces with characteristic frequencies of $25,50,100$, and $200 \mathrm{MHz}$, and broaden the bandwidth for analysis by summing the amplitude spectra of the four signals. I compute the spectrum at each reflecting interface using spectral decomposition via Short-Time Fourier Transform (STFT) with a relatively long 
time window length of $1.5 / 25 \mathrm{MHz}$. While there are wavelet based approaches that can provide higher time resolution, the long window STFT approximates a running average spectrum. This approach minimizes spectral anomalies due to small scale scattering in the vicinity of the reflecting interface and therefore is more robust for field data analysis.

For the layer 2 spectral ratio analysis I use the layer 1/layer 2 reflection for the reference spectrum and the layer 2/layer 3 reflection is then the target spectrum (Figure 3). In this and all subsequent cases, $\sigma_{\mathrm{dc}}$ and $\varepsilon_{\infty}$ are held constant at the known model values. In the noise free synthetic data, the estimated values for $\varepsilon_{\mathrm{dc}}$ and $\tau$ in layer 2 differ from the model values by $2 \%$ and $14 \%$ respectively.

The results above are encouraging, but the spectral ratio approach must be used carefully as large changes in the frequency dependence across the reflecting boundary can substantially alter the ratios. Consider model layer 3 where the reference spectrum is the layer 2/layer 3 reflection and the layer 3/layer 4 reflection is the target spectrum. The estimated parameters for layer 3 differ substantially from the model values (Table 1). This deviation is caused by a strong frequency dependence of the reflection coefficient at the layer 3/layer 4 boundary related to the large change in the dispersion curve (Bradford, 2007). Since there is only small variation in the shape of the dispersion curves across the layer 1/layer 2 and layer 2/layer 3 boundaries, frequency dependent reflection has only a minimal impact on the spectral ratios and the layer 2 parameter estimates are close to the model values.

Table 1: Model Debye parameters and inverted parameters for three cases -1 ) simple layered model with no noise, 2) $2 \%$ bandlimited noise added, 3 ) fine scale ( $2 \mathrm{~cm}$ thick layers) $2 \%$ random variability of permittivity and conductivity superimposed on the simple layered model.

\begin{tabular}{|c|c|c|c|c|c|c|c|c|c|c|c|}
\hline & \multicolumn{4}{|c|}{ Model parameters } & \multicolumn{5}{c|}{ Inverted parameters } \\
\cline { 2 - 13 } & \multicolumn{4}{|c|}{ Static parameters } & \multicolumn{2}{c|}{$\begin{array}{c}\text { Parameters to be } \\
\text { inverted }\end{array}$} & \multicolumn{2}{c|}{ No noise } & \multicolumn{2}{c|}{$\begin{array}{c}2 \% \text { Random } \\
\text { noise }\end{array}$} & \multicolumn{2}{c|}{$\begin{array}{c}\text { Scattering } \\
\text { model }\end{array}$} \\
\hline Layer & $\begin{array}{c}\text { Thickness } \\
(\mathrm{m})\end{array}$ & $\begin{array}{c}\sigma_{\mathrm{dc}} \\
(\mathrm{mS} / \mathrm{m})\end{array}$ & $\varepsilon_{\infty}$ & $\varepsilon_{\mathrm{dc}}$ & $\tau(\mathrm{ns})$ & $\varepsilon_{\mathrm{dc}}$ & $\begin{array}{c}\tau \\
(\mathrm{ns})\end{array}$ & $\varepsilon_{\mathrm{dc}}$ & $\tau(\mathrm{ns})$ & $\varepsilon_{\mathrm{dc}}$ & $\tau(\mathrm{ns})$ \\
\hline 1 & 4 & 6.00 & 25.6 & 29.0 & 22.2 & & & & & & \\
\hline 2 & 4 & 3.29 & 13.9 & 15.4 & 14.7 & 15.1 & 12.6 & 14.5 & 8.2 & 15.2 & 13.0 \\
\hline 3 & 4 & 4.19 & 17.4 & 19.6 & 17.0 & 15.5 & 6.0 & & & & \\
\hline 4 & $\infty$ & 42.0 & 35.0 & 43.0 & 18.3 & & & & & & \\
\hline
\end{tabular}

I repeated the inversion for two additional cases. First I added band limited random noise, with a maximum amplitude equal to $2 \%$ of the maximum amplitude in the data. The spectral ratio is sensitive to this noise and the estimated value of $\tau$ is lower than the model value by nearly a factor of 2 . Finally, to test the sensitivity to random heterogeneity within a given layer, I divided the model into $2 \mathrm{~cm}$ thick layers and introduced $\mathrm{a} \pm 2 \%$ random perturbation of the wavenumber into the background model. The spectral ratio method proved less sensitive to this scattering and parameter estimates are comparable to those computed using the noise free model (Table 1). In field data, both heterogeneity and random noise (and potentially coherent noise) will alter the spectra and the data and results must be evaluated carefully. 

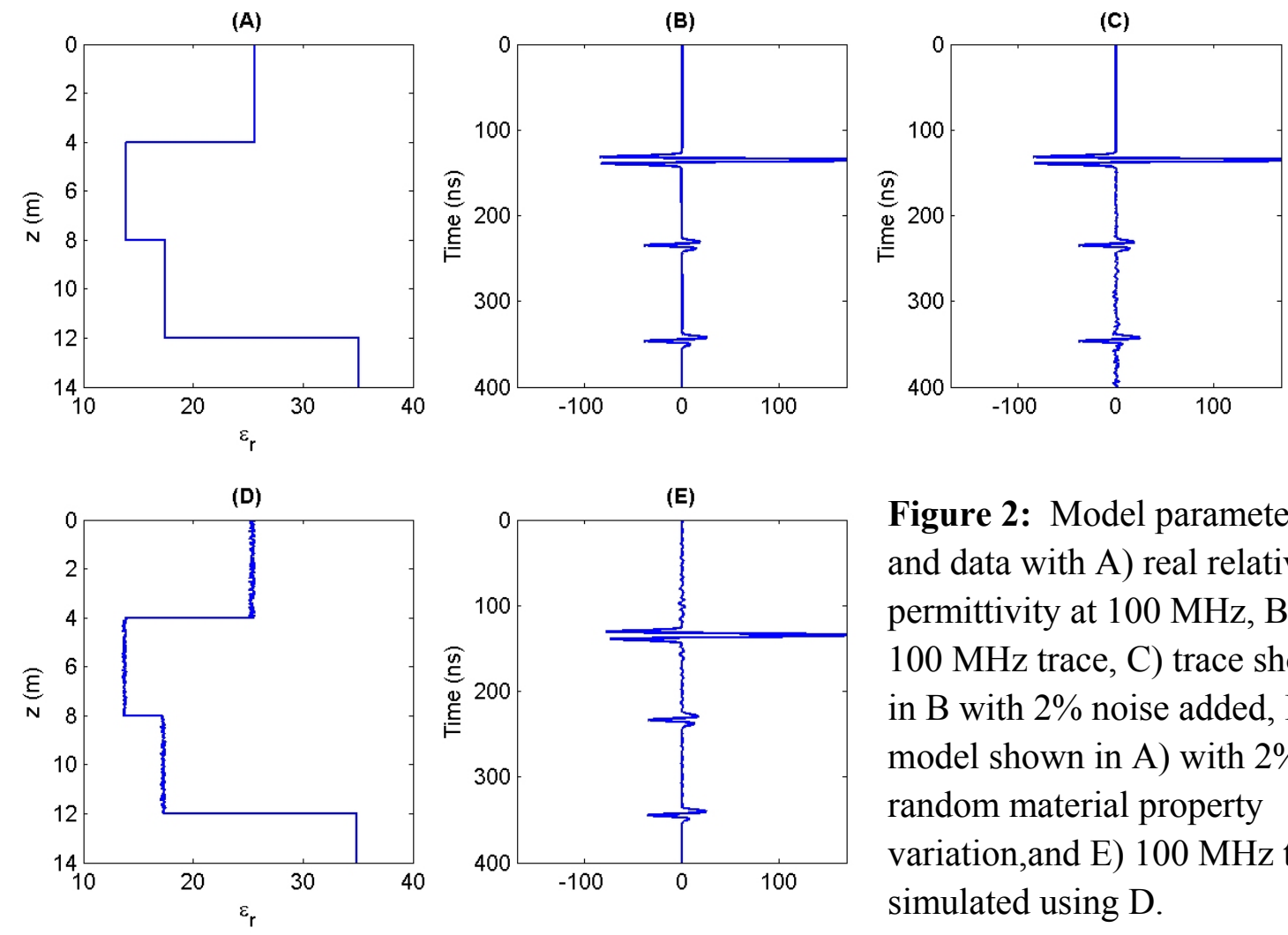

Figure 2: Model parameters and data with A) real relative permittivity at $100 \mathrm{MHz}, \mathrm{B}$ ) $100 \mathrm{MHz}$ trace, C) trace shown in B with $2 \%$ noise added, D) model shown in A) with $2 \%$ random material property variation, and E) $100 \mathrm{MHz}$ trace simulated using D.

(A)

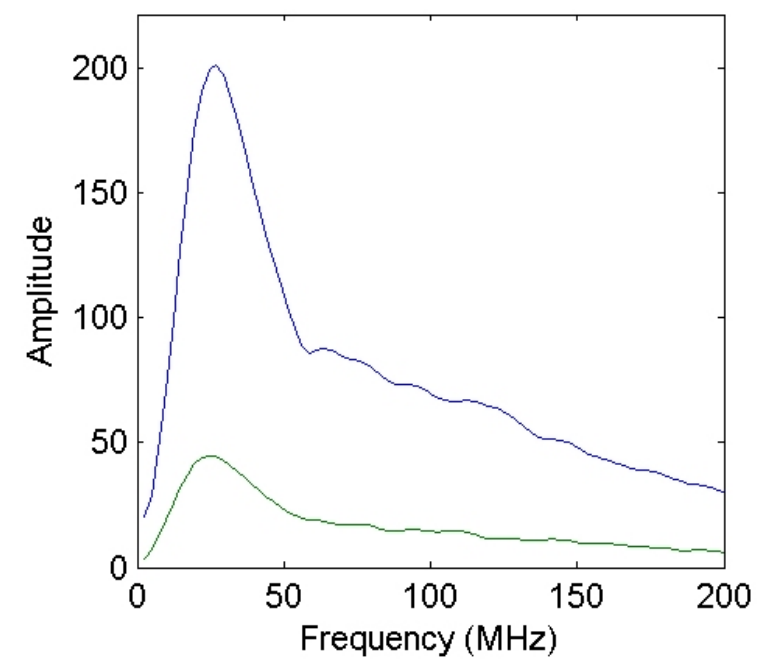

(B)

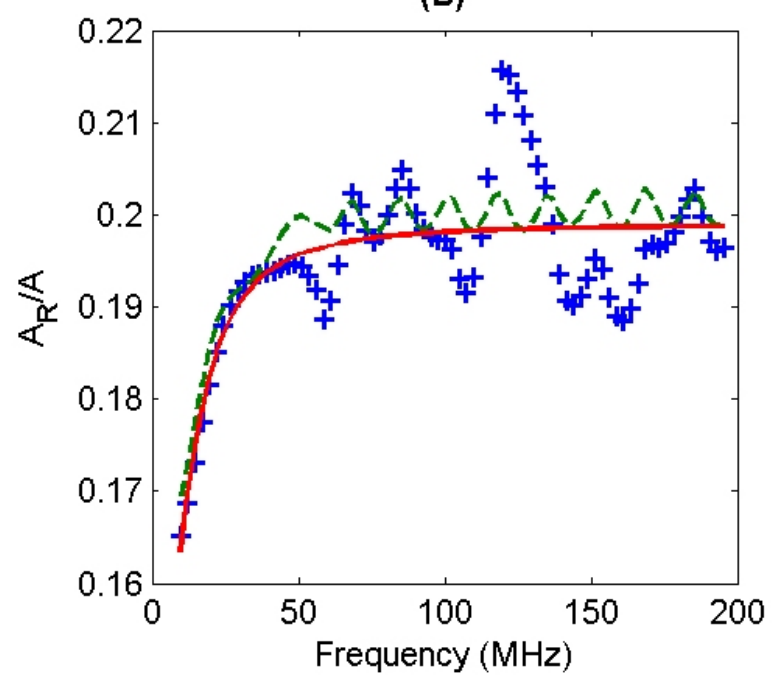

Figure 3: A) Summed 25, 50, 100, and $200 \mathrm{MHz}$ model reflection spectra used to compute spectral ratios. The high amplitude blue curve is the layer 1/layer 2 reference spectrum and the green curve is the layer 2/layer 3 spectrum. B) Spectral ratios for the noise free model (dashed) and scattering model (crosses). The solid red line is the inversion result for the scattering model. 


\section{Field Example: The Boise Hydrogeophysical Research Site}

The Boise Hydrogeophysical Research Site consists of a field scale experimental well field located in an unconfined sand and gravel aquifer adjacent to the Boise River approximately $12 \mathrm{~km}$ from downtown Boise. Stratigraphy within the aquifer is divided into five primary units based on porosity differences (Barrash and Reboulet, 2004), with Unit 1 being the deepest and Unit 5 the shallowest. The base of the aquifer is defined by a clay aquitard at a depth of $\sim 18 \mathrm{~m}$. Generally, Unit 5 consists of well sorted coarse grained sand, whereas Units 1-4 consist of poorly sorted sands gravels and cobbles. Detailed unit characteristics are given in Table 2.

Table 2: Properties of the 5 major stratigraphic units at the BHRS.

\begin{tabular}{|c|c|c|c|c|}
\hline Unit & $\sim$ Thickness & Mean Porosity & Porosity Variance & Dominant Composition \\
\hline 5 & $0-4 \mathrm{~m}$ & 0.429 & 0.003 & Coarse sand \\
\hline 4 & $1-5 \mathrm{~m}$ & 0.232 & 0.002 & Pebble/cobble dominated \\
\hline 3 & $\sim 3 \mathrm{~m}$ & 0.172 & 0.0006 & Pebble/cobble dominated \\
\hline 2 & $\sim 6 \mathrm{~m}$ & 0.243 & 0.002 & Pebble/cobble dominated \\
\hline 1 & $\sim 2 \mathrm{~m}$ & 0.182 & 0.0006 & Pebble/cobble dominated \\
\hline
\end{tabular}

The BHRS is the site of numerous previous borehole and surface GPR experiments (e.g. Bradford et al., 2008; Clement and Barrash, 2006; Clement et al., 2006; Clement and Knoll, 2006; Clement et al., 1999). Well defined radar reflections are observed at all major unit boundaries with the exception of the Unit 3/Unit 2 boundary which is gradational (Figure 4).

I designed a GPR field test to evaluate the spectral ratio method for determining non-linear attenuation parameters. To maximize the available bandwidth, the field team acquired coincident data with 25, 50, 100, and $200 \mathrm{MHz}$ antennas using a Sensors and Software Pulse Ekko Pro GPR system with $1000 \mathrm{~V}$ transmitter. All data were acquired with the same geometry to maintain the same travel path for all energy used in the analysis. The profile was $27 \mathrm{~m}$ long with $0.5 \mathrm{~m}$ spacing between traces. The source/receiver offset was set to $4 \mathrm{~m}$ to avoid transmitter/receiver coupling with the $25 \mathrm{MHz}$ antennas. The time sampling rate was $0.4 \mathrm{~ns}$ for all antennas to maintain spectral consistency and 32 traces were stacked for each antenna at each surface location.

After an initial spectral analysis to determine frequency content, bandpass filters were applied to each profile to attenuate noise above the maximum useable frequency in the data $(25 \mathrm{MHz}$ antennas, 1 2-50-100 MHz; $50 \mathrm{MHz}$ antennas, 1-2-100-200 MHz; $100 \mathrm{MHz}$ antennas, 1-2-200-400 MHz; $200 \mathrm{MHz}$ antennas, 1-2-250-500 MHz). $\mathrm{A} \mathrm{t}^{2}$ gain correction was applied to enhance deep reflection amplitudes. While the $100 \mathrm{MHz}$ antennas and $200 \mathrm{MHz}$ antennas produced a comparable maximum frequency (just below $100 \mathrm{MHz}$ ) the $200 \mathrm{MHz}$ antenna spectrum was broader but lower in amplitude than the $100 \mathrm{MHz}$ antenna spectrum. Further, the $100 \mathrm{MHz}$ antenna spectrum showed substantial variability that had a detrimental impact on the spectral analyses. This variability was not observed with any of the other antennas and the $100 \mathrm{MHz}$ data were not used for further analysis.

I manually picked time horizons at the water table, base of Unit 5, base of Unit 4, base of Unit 2, and base of Unit 1 as shown in Figure 4. Spectra were computed along each of these horizons with the STFT method and window described in the synthetic example above. I could not compute a suitable early time reference spectrum from the 25 and $50 \mathrm{MHz}$ data because the early arrivals were clipped.

Estimating $\varepsilon_{\mathrm{dc}}$ and $\tau$ required first estimating the two fixed parameters $\varepsilon_{\infty}$ and $\sigma_{\mathrm{dc}}$ for each unit. I assumed that the value of $\varepsilon$ measured by Clement and Knoll (2006) from $200 \mathrm{MHz}$ vertical radar profiles approximated $\varepsilon_{\infty}$. I used the average values of $\sigma_{\mathrm{dc}}$ reported by Oldenborger et al. (2007). 
Table 3: Debye parameter inversion results for the BHRS field data. $200 \mathrm{MHz}$ data were used for the Unit 4 and 5 inversions, and the combined 25, 50,200 MHz spectra were used for the Unit 2/3 inversion.

\begin{tabular}{|c|c|c|c|c|}
\hline & \multicolumn{4}{|c|}{ BHRS field data } \\
\hline & \multicolumn{2}{|c|}{ Static parameters } & \multicolumn{2}{c|}{ Inverted parameters } \\
\hline Unit & $\boldsymbol{\sigma}_{\mathbf{d c}}(\mathbf{m S} / \mathbf{m})$ & $\boldsymbol{\varepsilon}_{\infty}$ & $\boldsymbol{\varepsilon}_{\mathbf{d c}}$ & $\boldsymbol{\tau}(\mathbf{n s})$ \\
\hline 5 & 5.0 & 25.9 & $44 \pm 7$ & $10 \pm 3$ \\
\hline 4 & 1.0 & 14.0 & $20 \pm 5$ & $14 \pm 10$ \\
\hline $2 / 3$ & 1.0 & 12.8 & $16 \pm 2$ & $9 \pm 5$ \\
\hline
\end{tabular}

(A)

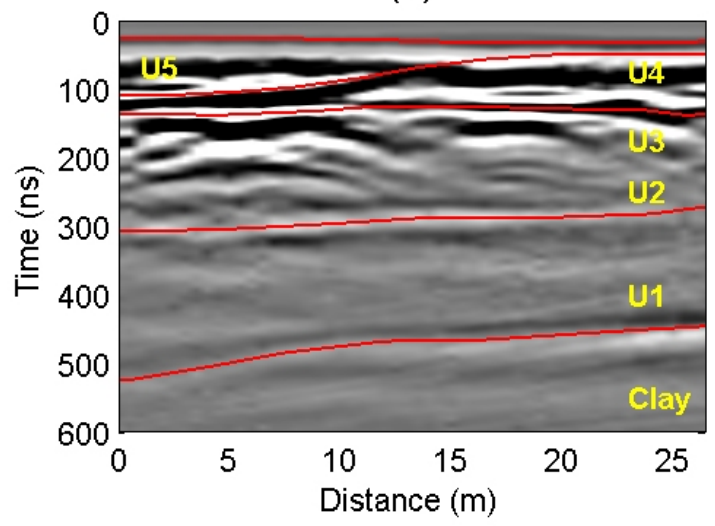

(C)

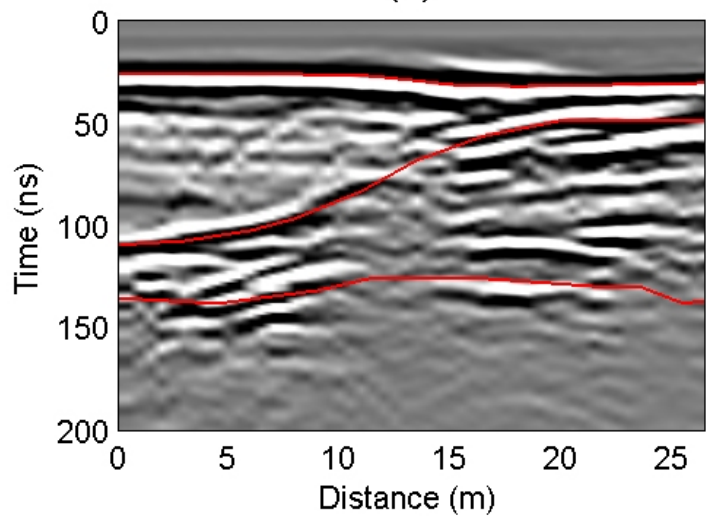

(B)

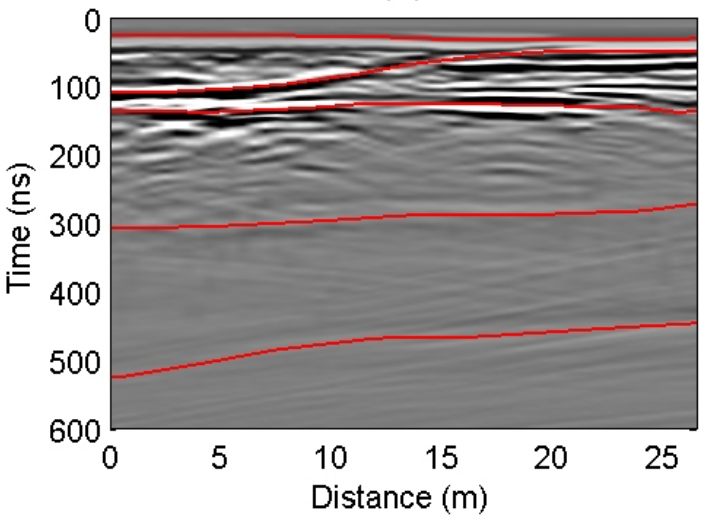

(D)

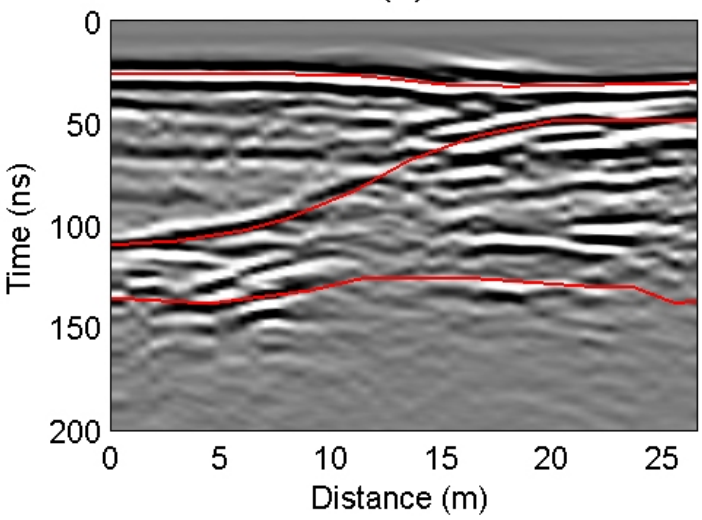

Figure 4: Field data from the BHRS experiment: A) $25 \mathrm{MHz}, \mathrm{B}) 50 \mathrm{MHz}, \mathrm{C}) 100 \mathrm{MHz}$, and D) 200 MHz. Units 1-5 (properties in Table 2) and the clay aquitard are noted in A). Red lines show horizons used for spectral analysis. The shallowest horizon is the water table reflection which dominates the radargram in $\mathrm{C}$ and $\mathrm{D}$ and is the interference of the direct wave and water table reflection in $\mathrm{A}$ and $\mathrm{B}$. All other horizons are at unit boundaries. Note that high frequency surface scattering from trees and bushes is evident in the $50 \mathrm{MHz}$ data below $300 \mathrm{Mhz}$. Only the upper $200 \mathrm{~ns}$ of the 100 and $200 \mathrm{MHz}$ profiles are shown to illustrate the resolution of the shallow section.

Because of the early-time difficulties with the 25 and $50 \mathrm{MHz}$ data, I utilized the $200 \mathrm{MHz}$ profiles only for the spectral ratio analysis of Units 4 and 5, and used the water table reflection as the reference spectrum (Figure 5A-D). Since Unit 5 pinches out near the center of the profile, I completed the Unit 5 analysis with the first $13 \mathrm{~m}$ of the profile, and the Unit 4 analysis with the last $14 \mathrm{~m}$ of the 

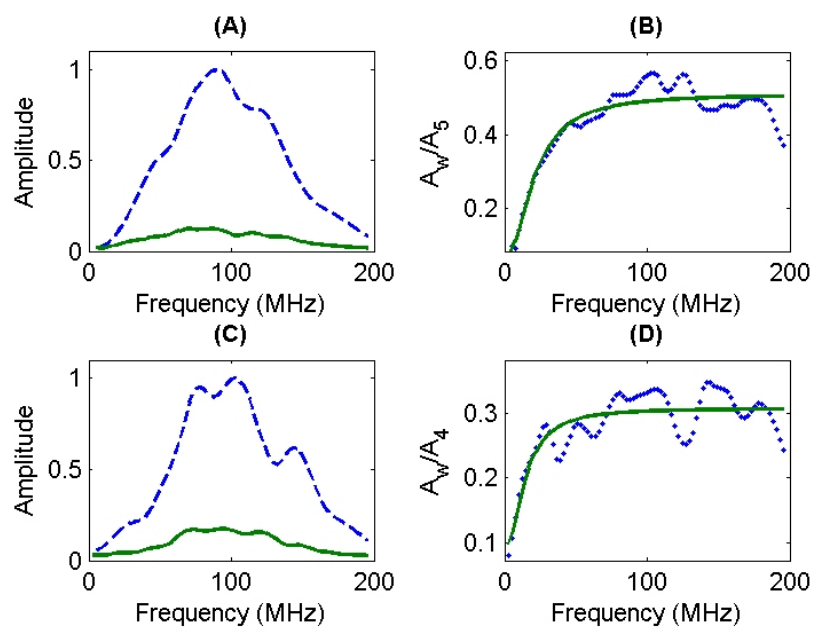

(E)
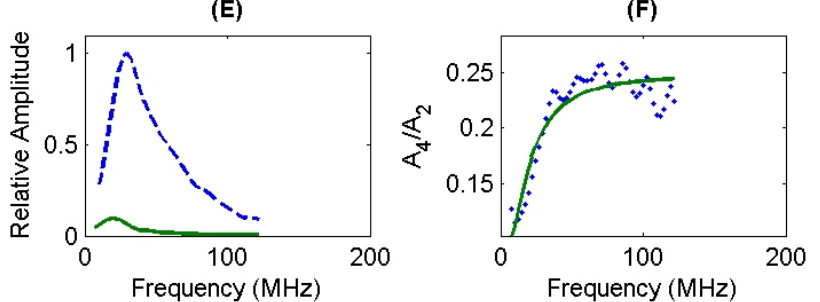

Figure 5: Mean reference (blue dashed) and target spectra (green solid) along with amplitude ratios and Debye models with parameters given in Table 3 . Unit 5 (A,B), Unit 4 (C, D), and Units $3 / 2$ (E,F). The spectral ratios for each unit closely follow a Debye relaxation model and the estimated relaxation times are within the range of laboratory measurements reported for sands.

profile. For these and all subsequent analyses, the parameter estimation was completed for every trace within the analysis area. The values reported in Table 3 are the means and standard deviations of these results.

Since there was no distinct reflection from the base of Unit 3 , the parameters estimated for the interval between the base of Unit 4 and Unit 2 represent effective values of the Unit 3/Unit 2 properties. For this analysis I summed the 25,50 , and $200 \mathrm{MHz}$ spectra (Figure 5E). I used the base of Unit 4 reflector as the reference and the base of Unit 2 as the target to compute the spectral ratios (Figure 5F). The entire $54 \mathrm{~m}$ profile was included in the computation. The base of Unit 2/base of Unit 1 spectral ratios did not produce useful results - that is, they did not follow a Debye relaxation spectrum. This may be due to the low signal to noise ratio along the base of Unit 1 reflection, or distortion of the ratio caused by frequency dependent reflection from the clay aquitard.

Results of the parameter inversions are shown in Table 3. The estimated relaxation times for Units 2 5 are within the range of laboratory measurements reported in the literature for saturated sands (e.g. Powers, 1997; Powers and Olhoeft, 1996). The high variability in Units $2 / 3$ and 4 is comparable to the variability caused by introduction of random noise in the synthetic example and the signal to noise for the reflections from the base of these units is substantially lower than that for Unit 5. The variability can likely be attributed in part to the high degree of heterogeneity that characterizes Units 1-4 (Table 2). It should be noted that the estimated relaxation times in all four units are within estimated uncertainty of each other and that a single relaxation time could describe the entire aquifer. The estimated values for $\varepsilon_{\mathrm{dc}}$ exhibit standard deviations of less than $25 \%$ for all units, and the estimates of 20 and 16 are comparable to laboratory measured values. The estimate of 44 in Unit 5 is larger than expected, but may be impacted by incorrect estimates of $\varepsilon_{\infty}$ and/or $\sigma_{\mathrm{dc}}$.

\section{Conclusions}

The method of spectral ratios is a promising approach to the estimation of frequency dependent attenuation parameters in GPR reflection data. Attenuation of reflected energy propagating through the each of the four upper units of the BHRS aquifer is well described with a single Debye relaxation mechanism. It must be recognized that the frequency dependent response observed at the surface is 
influenced by a number of factors in addition to the intrinsic attenuation including frequency dependent reflection and transmission and scattering. The estimated Debye parameters may be hydrologically relevant, however further research and laboratory measurements are required to to determine if there is a meaningful link.

\section{References}

Barrash, W., and E.C. Reboulet, 2004, Significance of porosity for stratigraphy and textural composition in subsurface coarse fluvial deposits, Boise Hydrogeophysical Research Site: Geological Society of America Bulletin, 116, 1509-1073, doi:10.1130/B25370.1.

Bradford, J.H., 1999, Characterizing shallow aquifers with wave-propagation based geophysical techniques: Imaging and attribute analysis: $\mathrm{Ph}$. D. thesis, Rice University.

Bradford, J.H., 2007, Frequency dependent attenuation analysis of ground-penetrating radar data: Geophysics, 72, J7-J16.

Bradford, J.H., W.P. Clement, and W. Barrash, 2008, Estimating porosity via ground-penetrating radar reflection tomography: A controlled 3D experiment at the Boise Hydrogeophysical Research Site: Water Resources Research, in press.

Cai, J., and G.A. McMechan, 1999, 2-D ray-based tomography for velocity, layer shape, and attenuation from GPR data: Geophysics, 64, 1579-1593.

Clement, W., and W. Barrash, 2006, Crosshole radar tomography in a fluvial aquifer near Boise, Idaho: Journal of Environmental and Engineering Geophysics, 11, 171-184.

Clement, W.P., W. Barrash, and M.D. Knoll, 2006, Reflectivity modeling of ground penetrating radar: Geophysics, 71, K59-K66.

Clement, W.P., and M.D. Knoll, 2006, Traveltime inversion of vertical radar profiles: Geophysics, 71, K67-K76.

Clement, W.P., M.D. Knoll, L.M. Liberty, P.R. Donaldson, P. Michaels, W. Barrash, and J.R. Pelton, 1999, Geophysical surveys across the Boise Hydrogeophysical Research Site to determine geophysical parameters of a shallow, alluvial aquifer: SAGEEP '99, Symposium on the Application of Geophysics to Environmental and Engineering Problems, Environmental and Engineering Geophysical Society, 399-408.

Debye, P.J., 1945, Polar Molecules, Dover Publishing Inc.

Irving, J.D., and R.J. Knight, 2003, Removal of wavelet dispersion from ground-penetrating radar data: Geophysics, 68, 960-970.

Liu, L., J.W. Lane, and Y. Quan, 1998, Radar attenuation tomography using the centroid frequency downshift method: Journal of Applied Geophysics, 40, 106-116.

Morlet, J., G. Arens, E. Fourgeau, and D. Giardi, 1982, Wave propagation and sampling theory - Part I: Complex signal scattering in multilayered media: Geophysics, 47, 203-221.

Nelder, J.A., and R. Mead, 1965, A simplex method for function minimization: The Computer Journal, 7, 308-313.

Oldenborger, G.A., M.D. Knoll, P.S. Routh, and D.J. LaBrecque, 2007, Time-lapse ERT monitoring of an injection/withdrawal experiment in a shallow unconfined aquifer: Geophysics, 72, F177F187.

Powers, M.H., 1997, Modeling frequency-dependent GPR: The Leading Edge, 16, 1657-1662.

Powers, M.H., and G.R. Olhoeft, 1996, Modeling the response of leaking, buried pipes: SAGEEP '95

Symposium on the Application of Geophysics to Environmental and Engineering Problems,

Environmental and Engineering Geophysical Society, Proceedings, 525-534.

Turner, G., 1994, Subsurface radar propagation deconvolution: Geophysics, 59, 215-223. 
Xu, T., and G.A. McMechan, 1997, GPR attenuation and its numerical simulation in 2.5 dimensions: Geophysics, 62, 403-414.

\section{Acknowledgements}

The US Environmental Protection Agency funded this work under Grant \# X-97008501-0. A special thanks goes to Shannon Murray, Emily Hinz, and Josh Nichols, for helping with field data acquisition. 